\title{
Análise retrospectiva da aplicação da escala de Braden Q em terapia intensiva pediátrica
}

\section{Retrospective analysis of the application of the Braden Q scale in pediatric intensive care Análisis retrospectivo de la aplicación de la escala de Braden Q en terapia
intensiva pediátrica}

Marcelli Cristine Vocci, , , Amanda Sayuri Sitoo Onary', Meire Cristina Novelli e Castro', Amanda Fabíola de Oliveira Spadotto ${ }^{1}$, Cassiana Mendes Bertoncello Fontes ${ }^{1}$

\section{ORCID IDS}

Vocci MC https://orcid.org/0000-0003-0029-139X

Onary ASS (iD) https://orcid.org/0000-0002-5667-494X Castro MCN (D) https://orcid.org/0000-0002-0590-4127 Spadotto AFO (D) https://orcid.org/0000-0003-3707-185X

Fontes CMB (D) https://orcid.org/0000-0002-6579-8637

\section{COMO CITAR}

Vocci MC; Onary ASS; Castro MCN; Spadotto AFO; Fontes CMB. Análise retrospectiva da aplicação da escala de Braden Q em terapia intensiva pediátrica. ESTIMA, Braz. J. Enterostomal Ther., 2020, 18: e2820. https://doi.org/10.30886/estima.v18.941_PT

\section{RESUMO}

Objetivo: caracterizar o perfil dos pacientes que desenvolveram lesão por pressão e analisar os escores retrospectivos obtidos na aplicação da Escala de Braden Q em terapia intensiva pediátrica. Método: estudo de coorte retrospectivo com análise de dados secundários de 34 prontuários eletrônicos de pacientes internados em terapia intensiva pediátrica. Resultados: da análise dos escores obtidos com aplicação da Escala de Braden Q, foram identificados seis registros de lesão por pressão; a média de idade foi de 8,1 anos; 66,6\% do sexo masculino; média de 41 dias de internação; principais diagnósticos estão relacionados a doenças do sistema respiratório, cardíaco e neurológico; todos utilizaram suporte de ventilação mecânica invasiva e apresentaram prescrição de jejum e uso de sonda nasogástrica. Conclusão: o perfil clínico está correlacionado ao sexo masculino, à maior frequência de doenças respiratórias com necessidade de ventilação mecânica, ao uso de drogas vasoativas, vasopressoras e sedativas, e à sonda como via de administração de dieta. Todos apresentaram escores de alto risco para desenvolvimento de lesão por pressão.

DESCRITORES: Lesão por pressão; Unidades de terapia intensiva pediátrica; Enfermagem de cuidados críticos; Estomaterapia.

1. Universidade Estadual Paulista Júlio de Mesquita Filho - Faculdade de Medicina de Botucatu - Departamento de Enfermagem - Botucatu (SP), Brasil.

*Autora correspondente: marcelli.vocci@unesp.br

Recebido: Ago. 24, 2020 | Aceito: Nov. 04, 2020 


\begin{abstract}
Objective: to characterize the profile of patients who developed pressure injuries and to analyze the retrospective scores obtained in the application of the Braden Q Scale in pediatric intensive care. Method: retrospective cohort study with analysis of secondary data from 34 electronic medical records of patients admitted to pediatric intensive care. Results: from the analysis of the scores obtained with the application of the Braden Q Scale, six records of pressure injuries were identified; the average age was 8.1 years; $66.6 \%$ were male; average of 41 days of hospitalization; main diagnoses are related to diseases of the respiratory, cardiac and neurological systems; all used invasive mechanical ventilation support and had a fasting prescription and use of a nasogastric tube. Conclusion: the clinical profile is correlated with the male gender, the higher frequency of respiratory diseases requiring mechanical ventilation, the use of vasoactive, vasopressor and sedative drugs, and the tube as a route of dietary administration. All of them had high risk scores for developing pressure injuries.
\end{abstract}

DESCRIPTORS: Pressure injury; Pediatric intensive care units; Critical care nursing; Stomatherapy.

\title{
RESUMEN
}

Objetivo: caracterizar el perfil de los pacientes que desarrollaron lesión por presión y analizar las puntuaciones retrospectivas obtenidas en la aplicación de la Escala de Braden Q en terapia intensiva pediátrica. Método: estudio de cohorte retrospectivo con análisis de datos secundarios de 34 historias clínicas electrónicas de pacientes internados en terapia intensiva pediátrica. Resultados: del análisis de las puntuaciones obtenidas con la aplicación de la Escala de Braden Q, se identificaron seis registros de lesión por presión; el promedio de edad fue de 8,1 años; 66,6 \% del sexo masculino; promedio de 41 días de internación; los principales diagnósticos se relacionan a enfermedades del sistema respiratorio, cardíaco y neurológico; todos utilizaron asistencia de ventilación mecánica invasiva y presentaron prescripción de ayuno y uso de sonda nasogástrica. Conclusión: el perfil clínico está correlacionado al sexo masculino, a la mayor frecuencia de enfermedades respiratorias que requieren ventilación mecánica, al uso de fármacos vasoactivos, vasopresores y sedantes y a la sonda como vía de administración de dieta. Todos presentaron puntuaciones de alto riesgo para el desarrollo de lesión por presión.

DESCRIPTORES: Lesión por presión; Unidades de terapia intensiva pediátrica; Enfermería de cuidados críticos; Estomaterapia.

\section{INTRODUÇÃO}

A institucionalização geralmente é uma situação incômoda para o indivíduo e sua família, com aspectos peculiares quando se trata de hospitalização infantil, fase fundamental em que se estabelecem as bases do desenvolvimento da criança. Trata-se, portanto, de um acontecimento inesperado, no qual a criança passa por situações incômodas, como se adaptar às normas e regras institucionais, à convivência com os profissionais da saúde, além da realização de exames e procedimentos dolorosos ${ }^{1-3}$.

Como evento adverso à institucionalização, surge a lesão por pressão (LP), que pode retardar a recuperação, prolongar o tempo de internação, aumentar os custos hospitalares e a carga de trabalho dos profissionais, além de associar-se à taxa de mortalidade e ser um evento traumático, cujas lesões deixam cicatrizes e uma história no corpo da criança ${ }^{4,5}$.

Embora a literatura possua ampla publicação sobre o desenvolvimento de LP em adultos, estudos com pacientes pediátricos são escassos, pois as condições anatômicas e fisiopatológicas do desenvolvimento infantil, o tempo de internação prolongado, o déficit de mobilidade física, além das novas tecnologias adaptadas ao contexto da pediatria intensiva colocam o paciente pediátrico em risco de desenvolvimento de $\mathrm{LP}^{6,7}$.

A susceptibilidade ao desenvolvimento de LP pode ser avaliada por instrumentos de predição de risco, os quais direcionam para a utilização de medidas preventivas ${ }^{8}$. Um dos instrumentos mais conhecidos e utilizados em hospitais para auxiliar na identificação do risco de desenvolvimento de LP em pacientes pediátricos é a Escala de Braden Q (EB-Q), desenvolvida em 2004, a partir da versão para pacientes adultos 9 , e adaptada em 2011, transculturalmente, para uso no Brasil ${ }^{10}$.

Atualmente, a prevenção da LP é um aspecto desafiador para os enfermeiros e fundamenta-se na diminuição ou na eliminação de riscos passíveis de intervenções, sendo difícil prevenir e avaliar a eficácia de qualquer medida preventiva sem o conhecimento e a predição dos riscos de cada paciente. Com isso, o desenvolvimento da LP é um fenômeno multifatorial e extremamente complexo, podendo ter uma prevenção mais efetiva quando se identificam os fatores de maior influência para o seu desenvolvimento ${ }^{6}$.

Dessa forma, a realização deste estudo sobre a importância de instrumentos preditivos e o conhecimento do perfil de pacientes pediátricos de alto risco ou acometidos pela LP poderá contribuir para a obtenção de dados relevantes 
sobre o tema pouco estudado, uma vez que possibilitará o dimensionamento das características e das necessidades dessa clientela, servindo de subsídio para a otimização de recursos humanos e materiais na prevenção das lesões.

\section{OBJETIVO}

Caracterizar o perfil dos pacientes que desenvolveram LP e analisar os escores retrospectivos obtidos na aplicação da EB-Q em unidade de terapia intensiva pediátrica (UTIP).

\section{MÉTODO}

Estudo de coorte retrospectivo realizado a partir da análise de dados secundários obtidos do prontuário eletrônico de pacientes internados na UTIP de um hospital público.

O período retrospectivo da coleta de dados foi de dezembro de 2015 a agosto de 2018. Os critérios de elegibilidade foram: registro completo e preenchimento correto de, no mínimo, três avaliações sequenciais da Braden Q.Assim, foram incluídos na amostra final o total de 34 prontuários.

\section{Escala de Braden Q}

A Braden Qé composta por 7 subescalas, as quais pontuam escores de 1 a 4 . O escore final varia entre 7 e 28 pontos. Quanto menor o valor, maior será o comprometimento apresentado e, consequentemente, maior a exposição ao risco.

De acordo com os critérios da escala, categoriza-se o escore em duas faixas de risco geral, cut-off 22, sendo alto risco de desenvolvimento de LP quando escore final < 22; e baixo risco de desenvolvimento de LP quando escore final $\geq 22$.

Foram consideradas como variáveis quantitativas do estudo aquelas que compõem a Braden Q: mobilidade, atividade, percepção, umidade, fricção, nutrição, perfusão tecidual e oxigenação.

\section{Coleta e análise de dados}

A coleta de dados teve início em agosto de 2018, após a aprovação pelo Comitê de Ética em Pesquisa CAEE 40752115.3.0000.5411, e encerrou-se em outubro de 2018. Os dados foram solicitados formalmente ao núcleo responsável pela manutenção e controle do Sistema de Prontuário Eletrônico, e uma listagem dos pacientes internados na terapia intensiva pediátrica no período de dezembro de 2015 a agosto de 2018 foi fornecida. A partir dessa lista, a instituição disponibilizou um computador com acesso aos prontuários para análise detalhada das variáveis de interesse.

Os dados foram analisados por um profissional estatístico, obtendo a estatística descritiva dos dados, com frequência e as porcentagens para as variáveis qualitativas; e média, desvio padrão, mediana, mínima e máxima para as variáveis quantitativas.

\section{RESULTADOS}

No período retrospectivo eleito para a coleta dos dados, 34 pacientes compuseram a amostra do estudo. O perfil geral da amostra (Tabela 1) caracterizou-se pela predominância do sexo masculino 23 (67,65\%), média de 4,40 anos de idade e os diagnósticos mais frequentes estavam relacionados às doenças respiratórias 12 (35,29\%), cardíacas 10 (29,41\%), neurológicas $6(17,65 \%)$ e neoplásicas $6(17,65 \%)$.

Tabela 1. Caracterização do perfil da amostra. Botucatu (SP), Brasil - 2019.

\begin{tabular}{|c|c|}
\hline Variáveis & UTIP* \\
\hline Idade & n (\%) \\
\hline$<1$ ano & $17(50,00)$ \\
\hline $1-5$ anos & $4(11,76)$ \\
\hline $6-10$ anos & $7(20,59)$ \\
\hline $11-15$ anos & $6(17,65)$ \\
\hline Total & $34(100,00)$ \\
\hline Sexo & n (\%) \\
\hline Masculino & $23(67,65)$ \\
\hline Feminino & $11(32,35)$ \\
\hline Total & $34(100,00)$ \\
\hline Diagnósticos & n (\%) \\
\hline Respiratórias & $12(35,29)$ \\
\hline Cardíacas & $10(29,41)$ \\
\hline Neurológicas & $6(17,65)$ \\
\hline Neoplásicas & $6(17,65)$ \\
\hline Total & $34(100,00)$ \\
\hline
\end{tabular}

* UTIP: unidade de terapia intensiva pediátrica 
A aplicação da Braden Q gerou um total de 169 avaliações durante o período retrospectivo do estudo. Obtiveram-se médias de 27 avaliações por paciente, com mínima de 13 e máxima de 39 avaliações; a média dos escores totais foi de 14, demonstrando que 34 (100\%) pacientes estavam em alto risco de desenvolvimento de $\mathrm{LP}(<22)$ em relação ao cut-off atribuído pela escala.

As subescalas de Braden Qque apontaram menor porcentagem total no escore, ou seja, maior comprometimento foram: atividade, em $76 \%$ das avaliações; mobilidade, em 50\%; fricção e cisalhamento, em 32\%. A subescala que obteve menor média de escore individual das 169 avaliações realizadas foi a subescala "atividade", com média de 1,3 (desvio padrão $\pm 0,96$ ) de escore. A melhor avaliação individual, ou seja, a melhor média de escore entre as avaliações foi a subescala "umidade", com média de 2,8 (desvio padrão $\pm 0,88$ ).

Dos 34 prontuários analisados, em 6 (17\%) havia registro de desenvolvimento de LP. O perfil desses pacientes foi caracterizado como (Tabela 2): média de idade de 8,1 anos; 4 (66,67\%) do sexo masculino; média de 41 dias de internação, mínimo de 11 dias e máximo de 65 dias. Todos os pacientes com registro de desenvolvimento de LP foram submetidos à ventilação mecânica invasiva e à sonda vesical de demora; as drogas vasoativas e sedativas foram utilizadas em $100 \%$ daqueles que desenvolveram LP; para a variável nutrição, 6 (100\%) pacientes tiveram mais que dois registros de prescrição de jejum em algum momento durante o período de internação, sendo que $4(66,67 \%)$ apresentaram prescrição de nutrição parenteral e $2(33,33)$, prescrição de dieta enteral via sonda nasogástrica.

Com relação à caracterização e à classificação das lesões identificadas nos registros dos prontuários, o local mais acometido foi a região dos calcâneos, com $4(66,7 \%)$ lesões, e a região sacral, com $2(33,3 \%)$, sendo $3(50 \%)$ no estágio 2 e 3 no estágio 3 (50\%) de comprometimento tissular. As coberturas mais utilizadas foram o hidrocoloide, 3 (50\%) e papaína, 3 (50\%).

$\mathrm{O}$ registro de óbito foi identificado em quatro dos seis pacientes que apresentaram desenvolvimento de LP.

\section{DISCUSSÃO}

O desenvolvimento da LP relaciona-se com as características e as condições clínicas dos pacientes associadas às particularidades do cuidado prestado, revelando-se, portanto, como um problema multifatorial.
Tabela 2. Caracterização do perfil dos pacientes que desenvolveram lesão por pressão. Botucatu (SP), Brasil - 2019.

\begin{tabular}{|c|c|}
\hline Idade & n (\%) \\
\hline$<1$ ano & $3(50,00)$ \\
\hline $1-5$ anos & $0(00,00)$ \\
\hline $6-10$ anos & $2(33,33)$ \\
\hline $11-15$ anos & $1(16,67)$ \\
\hline Total & $6(100,00)$ \\
\hline Sexo & n (\%) \\
\hline Masculino & $4(66,67)$ \\
\hline Feminino & $2(33,33)$ \\
\hline Total & $6(100,00)$ \\
\hline Diagnósticos & n (\%) \\
\hline Respiratórias & $2(33,33)$ \\
\hline Cardíacas & $2(33,33)$ \\
\hline Neurológicas & $2(33,33)$ \\
\hline Total & $6(100,00)$ \\
\hline Dias de internação & $\mathrm{n}(\%)$ \\
\hline $1-30$ dias & $2(33,33)$ \\
\hline $31-65$ dias & $4(66,67)$ \\
\hline Total & $6(100,00)$ \\
\hline Administração da dieta & n (\%) \\
\hline Via enteral & $2(33,33)$ \\
\hline Via parenteral & $4(66,67)$ \\
\hline Total & $6(100,00)$ \\
\hline
\end{tabular}

O julgamento clínico do enfermeiro, fundamentado pelo conhecimento científico e pela experiência prática, quando aliado a instrumentos que permitam mensurar o risco para o desenvolvimento da lesão de forma objetiva, pode tornar o processo preventivo mais efetivo ${ }^{11}$.

A EB-Q demonstrou ser um instrumento eficaz e confiável com acurácia para predizer o risco de desenvolvimento de LP na população pediátrica, reiterando os achados de outros estudos ${ }^{6,12}$, nos quais os pacientes que tiveram escores mais críticos durante as avaliações, precisamente, desenvolveram o agravo. 
Um dos fatores de risco para o desenvolvimento da LP é o tempo de institucionalização, sendo que quanto maior a permanência do paciente sob cuidados intensivos, mais propensos estão ao desenvolvimento desse agravo ${ }^{13}$. A média de dias de internação dos pacientes que desenvolveram o agravo foi de 41, ou seja, maior do que os demais pacientes que não foram acometidos (23 dias).

Em outros estudos ${ }^{14-15}$, os achados identificados em relação aos diagnósticos de maior frequência corroboram os dados relacionados às doenças respiratórias identificados neste estudo. Sabe-se que as morbidades desse tipo de causa de internação em UTIP interferem negativamente, prejudicando o aporte de oxigênio para os tecidos, isto é, facilitando o desenvolvimento da LP.

Alguns medicamentos utilizados durante a internação, principalmente os de uso contínuo, podem colaborar para o desenvolvimento da LP, como as drogas vasoativas e vasopressoras. Devido à instabilidade hemodinâmica, essas classes medicamentosas são necessariamente prescritas, podendo predispor o desenvolvimento de LP por interferirem no fluxo sanguíneo periférico e na perfusão tissular ${ }^{16-17}$. A sedação e a analgesia em terapia intensiva colaboram no tratamento do paciente em estado grave, melhorando o desconforto respiratório e a adaptação à ventilação mecânica invasiva. Contudo, a sedação está associada ao déficit de mobilidade do paciente, prolongamento do tempo em ventilação mecânica e aumento das taxas de incidência de $\mathrm{LP}^{18-19}$.

Um estudo ${ }^{19}$ apontou a LP como o evento adverso mais notificado, tanto em pacientes com sedação profunda quanto leve, mostrando que $20 \%$ dos casos de LP estavam relacionados ao déficit sensorial ocasionado pelas medicações sedoanalgésicas. Assim, o enfermeiro deve estar apto a identificar esse grupo, implementando ações direcionadas que possam reduzir a incidência desse agravo e outras complicações.

Pacientes graves normalmente estão em uso de suporte ventilatório, sendo, portanto, um fator de risco importante devido à dificuldade em manter sua perfusão e uma oxigenação adequada por maior tempo, além disso, muitas vezes o suporte ventilatório dificulta a mudança de decúbito e a mobilização do paciente em virtude da sua instabilidade hemodinâmica, favorecendo o desenvolvimento da lesão $\mathrm{o}^{20-21}$.

O estado nutricional tem grande importância sobre as respostas fisiológicas do organismo. Durante a análise dos dados sobre as condições dos pacientes que desenvolveram o agravo, observou-se que a forma de administração de dieta mais utilizada foi via sonda nasogástrica, e o jejum foi prescrito para todos os pacientes. Frente a isso, o consumo alimentar inadequado surge como uma das principais causas e está diretamente relacionado ao processo de instalação da lesão. Situações que modifiquem o modo como o paciente se alimenta, alterando o processo digestivo, podem comprometer o processo fisiológico de absorção de nutrientes e seu adequado aproveitamento para importantes mecanismos fisiológicos do organismo $22-23$.

Entre as subescalas, a "atividade" obteve menor e maior pontuação individual e foi a que mais influenciou no desenvolvimento da LP; a subescala "umidade" foi a que menos influenciou no desenvolvimento da LP. Sabidamente, os pacientes acamados e/ou com atividade reduzida estão sujeitos ao fator pressão, que desempenha papel-chave no desenvolvimento do agravo ${ }^{14}$. Neste estudo, a falta de atividade (subescala atividade) foi um dos principais fatores de risco para o desenvolvimento do agravo, isso ocorre devido à incapacidade do indivíduo se mover, alternando posições no leito para proporcionar alívio da pressão em áreas vulneráveis do corpo.

A subescala que quantificou o fator umidade como contributivo para o desenvolvimento de LP apresentou maior escore, o que caracteriza a qualidade do cuidado, ou seja, a equipe de enfermagem tem como foco de cuidado e protocolo interno a frequência das trocas de fraldas a cada duas horas.

Quando se identifica o desenvolvimento de uma lesão, torna-se essencial sua classificação para escolha adequada da cobertura a ser utilizada ${ }^{24-25}$. No presente estudo, os estágios 2 e 3 de LP foram identificados com maior frequência e, em decorrência disso, as coberturas mais utilizadas foram o hidrocoloide e a papaína. $\mathrm{O}$ hidrocoloide também pode ser utilizado como medida preventiva em regiões de maior risco de desenvolvimento do agravo, sendo que os danos possivelmente causados pela fricção podem ser reduzidos pelo uso dessa barreira de proteção. Já a cobertura de papaína tem indicação nas lesões de estágio 3 e 4 e nas lesões que possuem tecidos desvitalizados, proporcionando ação bactericida, desbridamento químico, ação anti-inflamatória e diminuição de edema local ${ }^{24-25}$.

Como limitação, este estudo apresenta quantidade restrita de participantes devido à unidade abordada possuir apenas sete leitos ativos e baixa rotatividade, não sendo possível, assim, generalizar os dados, deixando evidente a 
necessidade de estudos que estabeleçam dados referentes ao conhecimento do fenômeno LP em pacientes críticos pediátricos, ainda pouco estudados.

\section{CONCLUSÃO}

O perfil clínico caracterizou-se por indivíduos do sexo masculino, com maior frequência de doenças respiratórias com necessidade de ventilação mecânica; uso de drogas vasoativas, vasopressoras e sedativas; e uso de sonda nasogástrica ou nasoenteral como principal via de administração de dieta. Todos os pacientes apresentaram escores de alto risco para desenvolvimento de LP. Assim, o presente estudo trouxe a descrição do perfil de risco dos pacientes pediátricos vulneráveis ao desenvolvimento do agravo como forma de vigilância e alerta para a necessidade de conscientização das instituições e dos profissionais da saúde acerca da importância da avaliação de risco e do planejamento individualizado de ações preventivas, possibilitando que essas medidas sejam direcionadas de acordo com o risco de cada paciente, otimizando os recursos humanos e materiais.

\section{AGRADECIMENTOS}

Os autores gostariam de agradecer ao $\mathrm{CNPq} / \mathrm{PROPe} /$ UNESP pela bolsa PIBIC reitoria edital 2018/2019.

\section{CONTRIBUIÇÃO DOS AUTORES}

Conceitualização: Vocci MC, Fontes CMB; Metodologia: Vocci MC, Fontes CMB; Investigação: Vocci MC, Onary ASS; Redação - Primeira Versão: Vocci MC, Onary ASS, Fontes CMB; Aquisição de Financiamento: Fontes CMB; Recursos: Fontes CMB, Vocci MC, Onary ASS; Supervisão: Fontes CMB.

\section{REFERÊNCIAS}

1. Costa TS, Morais AC. Child hospitalization: child living from graphical representations. Rev Enferm UFPE on line 2017; [citado 2020 jun 18];11(Supl1):358-67. Disponível em: https://periodicos.ufpe.br/revistas/revistaenfermagem/ article/view/11916

2. Gomes GLL, Fernandes MGM, Nóbrega MML. Ansiedade da hospitalização em crianças: análise conceitual. Rev Bras Enferm 2016;69(5):940-5. https://doi.org/10.1590/00347167-2015-0116

3. Santos GS, Pieszak GM, Gomes GC, Biazus CB, Silva SO Contributions of better childhood for growth and child development in family perception. Rev Pesqui Cuid Fundam. 2019; [citado 2020 jun 18];11(1):67-73. Disponível em: http://www.seer.unirio.br/index.php/cuidadofundamental/ article/viewFile/6465/pdf_1

4. Matozinhos FP, Velasquez-Melendez G, Tiensoli SD, Moreira AD, Gomes FSL. Factors associated with the incidence of pressure ulcer during hospital stay. Rev Esc Enferm USP 2017;51:e03223. https://doi.org/10.1590/s1980$220 \times 2016015803223$

5. Ferreira MKM, Gurgel SS, Lima FET, Cardoso MVLML, Silva VM. Instruments for the care of pressure injury in pediatrics and hebiatrics: an integrative review of the literature. Rev Latino-Am Enfermagem 2018;26:e3034. https://doi. org/10.1590/1518-8345.2289.3034
6. Vocci MC, Fontes CMB, Abbade LPF. Pressure injury in the pediatric population: cohort study using the Braden Q scale. Adv Skin Wound Care 2018;31(10):456-61. https://doi. org/10.1097/01.ASW.0000542529.94557.0a

7. Rodrigues CBO, Prado TN, Nascimento LCN, Laignier MR, Primo CC, Bringuente MEO. Management tools in nursing care for children with pressure injury. Rev Bras Enferm 2020;73(supp/4):e20180999. https://doi.org/10.1590/00347167-2018-0999

8. VasconcelosJMB, Caliri MHL. Nursing actions before and after a protocol for preventing pressure injury in intensive care. Esc Anna Nery 2017; [citado 2020 out 29];21(1):e20170001. Disponível em: https://www.scielo.br/pdf/ean/v21n1/14148145-ean-21-01-e20170001.pdf

9. Curley MAQ, Razmus IS, Roberts KE, Wypij D. Predicting pressure ulcer risk in pediatric patients: the Braden Q scale. Nurs Res 2003;52(1):22-33. https://doi. org/10.1097/00006199-200301000-00004

10. Maia ACAR, Pellegrino DMS, Blanes L, Dini GM, Ferreira LM. Portuguese translation and validation of the Braden Q scale for predicting pressure ulcer risk in pediatric patients. Rev Paul Pediatr 2011;29(3):406-14. https://doi.org/10.1590/ S0103-05822011000300016

11. Souza MFC, Zanei SSV, Whitaker IY. Risk of pressure injury in the ICU: transcultural adaptation and reliability of 
EVARUCI. Acta Paul Enferm 2018;31(2):201-8. https://doi. org/10.1590/1982-0194201800029

12. Vocci MC, Toso LAR, Fontes CMB. Application of the Braden Q scale at a pediatric intensive care unit. Rev enferm UFPE on line. 2017; [citado 2020 mai 8];11(1):165-72. Disponível em: https://periodicos.ufpe.br/revistas/revistaenfermagem/ article/view/11890/14356

13. Campanili TCGF, Santos VLCG, Strazzieri-Pulido KC, Thomaz PBM, Nogueira PC. Incidence of pressure ulcers in cardiopulmonary intensive care unit patients. Rev Esc Enferm USP 2015;49(Esp):7-14. https://doi.org/10.1590/ S0080-623420150000700002

14. Curley MAQ, Hasbani NR, Quigley SM, Stellar JJ, Pasek TA, Shelley SS et al. Predicting pressure injury risk in pediatric patients: the Braden QD scale. J Pediatr 2018;192(2):189-95. https://doi.org/10.1016/j.jpeds.2017.09.045

15. Smith HA, Moore Z, Tan MH. Cohort study to determine the risk of pressure ulcers and developing a care bundle within a paediatric intensive care unit setting. Intensive Crit Care Nurs 2019;53:68-72. https://doi.org/10.1016/j. iccn.2019.04.008

16. Cox J, Roche S. Vasopressors and development of pressure ulcers in adult critical care patients. Am J Crit Care 2015;24(6):501-10. https://doi.org/10.4037/ajcc2015123

17. Tallo FS, Vendrame LS, Lopes RD, Lopes AC. Invasive mechanical ventilation in the emergency room: a review for clinicians. Rev Bras Clin Med 2013; [citado 2020 jun 22];11(1):48-54. Disponível em: http://files.bvs.br/ upload/S/1679-1010/2013/v11n1/a3395.pdf

18. Santos $L R C L$, Lino AIA. Risks of pressure injury: application of the Braden scale in intensive care. ESTIMA, Braz J Enterostomal Ther 2018;16:e0818. https://doi. org/10.30886/estima.v16.443_PT
19. Barbosa TP, Beccaria LM, Silva DC, Bastos AS. Association between sedation and adverse events in intensive care patients. Acta Paul Enferm 2018,31(2):194-200. https://doi. org/10.1590/1982-0194201800028

20. Loudet $\mathrm{Cl}$, Marchena MC, Maradeo MR, Fernández SL, Romero MV, Valenzuela GE et al. Reducing pressure ulcers in patients with prolonged acute mechanical ventilation: a quasiexperimental study. Rev Bras Ter Intensiva 2017; [citado 2020 jun 20];29(1):39-46. Disponível em: https://www.scielo.br/pdf/ rbti/v29n1/0103-507X-rbti-29-01-0039.pdf

21. Miske LJ, Stetzer M, Garcia M, Stellar JJ. Airways and injuries: protecting our pediatric patients from respiratory device-related pressure injuries. Crit Care Nurs Clin North Am 2017;29(2):187-204. https://doi.org/10.1016/j. cnc.2017.01.006

22. Ministério da Saúde (BR). Manual de terapia nutricional na atenção especializada hospitalar no âmbito do Sistema Único de Saúde - SUS. $1^{a}$ ed. Brasília (DF): Ministério da Saúde; 2016.

23.S imony RF, Chaud DMA, Abreu ES, Blascovi-Assis SM. Nutritional status of neurological patients with reduced mobility. J Hum Growth Dev. (Online) 2014; [citado 2020 jul 1];24(1):42-48. Disponível em: http://pepsic.bvsalud.org/ $\mathrm{pdf} / \mathrm{rbcdh} / \mathrm{v} 24 \mathrm{n} 1 / 06 . \mathrm{pdf}$

24. Vocci MC, Abbade LPF, Fontes CMB. Protocolo preventivo: lesão por pressão em unidades pediátricas. Botucatu: Universidade Estadual Paulista "Júlio de Mesquita Filho", Faculdade de Medicina, 2016; [citado 2020 jul 1]; $1^{\text {a }}$ ed. Disponível em: http://www.hcfmb.unesp.br/wp-content/ uploads/2015/09/Protocolo.pdf

25. National pressure ulcer advisory panel (NPIAP). Pressure injury stages. [Internet]. 2016 [citado 2020 jul 1]. Disponível em: https://npiap.com/page/PressurelnjuryStages 